\title{
Head and neck radiotherapy with tele-cobalt machine- efficacy and need for tissue compensation
}

\begin{abstract}
External Beam Radiotherapy (EBRT) remains the mainstay for radical treatments, in malignancies in head and neck regions. Head and Neck malignancies form around $50 \%$ of radiotherapy patients in the north eastern parts of the Indian subcontinent. Because of less inter-field separations, the penetration requirements of the treatment plans makes the telecobalt beam sufficient for executing radiotherapy, with proper immobilization and use of wedges if required. Inter-field thickness variations and irregular contour affect uniformity in delivered doses, and adverse reactions are encountered because of skewed isodose curves seriously affecting the volume dose variations in treatment planning. Normal tissue reactions affect effectiveness of the treatment and therefore deter the Quality of Life (QOL).
\end{abstract}

EBRT with a telecobalt machine (Theratron 780C) started in 2006 and completed a decade at our centre. About 15 patients per day receive radiotherapy for head and neck treatments. To overcome skin reactions and excess dose due to small contour of neck, a need was felt to introduce tissue compensation. Ellis type tissue compensation using $1 \mathrm{~cm}$ thick Aluminum metal is introduced. The tissue deficiency is measured by an L shaped gridded type Lucite frame work. For individual patients the custom type compensator tray is prepared on a Lucite plate, mountable in the shadow tray of the cobalt machine. The attenuation coefficient of metal rods $\mu=0.1462 \mathrm{~cm}^{-1}(1 \mathrm{~cm}$ $\mathrm{Al}=2.57 \mathrm{~cm}$ water). A simple type of POP immobilization is prepared in place of thermoplastic immobilization shell. The Lucite mounting tray has attenuation about $4 \%$.

Lateral opposing fields with compensation reduced resultant iso doses $180 \%$ and $170 \%$ in neck and chin levels to $150 \%$ with uniform tumor volume homogeneous dose of $160 \%$. Portal radiographs with and without compensator showed uniform tissue irradiation, also a method to verify field positions. If this method is applied to all the head and neck radiotherapy patients both for AP-PA and Lateral fields, the morbidity up to epidermis, and overdose effects could be brought down, making tele cobalt treatments more effective. In the era of high technology radio-therapy, these types of innovations at hospital level promote cost-effective radical treatments to cancer patients, because cancer care all over the globe becomes unaffordable.

Keywords: tissue compensation, telecobalt, head and neck radiotherapy, skin reactions, patients, cancer, treatments, compensator, homogeneous, overdose, immobilization, isodose, quality
Volume 2 Issue 3 - 2017

\author{
Ramamoorthy Ravichandran, Chinnaraj \\ Manimegalai \\ Department of Radiation Oncology, India
}

Correspondence: Ramamoorthy Ravichandran, Head, Medical Physics Unit, Department of Radiation Oncology, Cachar Cancer Hospital \& Research Center, Silchar-788 015,Assam, India, Tel 913842240608,919738174354 , Email ravichandranrama@rediffmail.com

Received: September 23, 2016 | Published: March 01, 2017
Abbreviations: EBRT, external beam radiotherapy; QOL, quality of life; IMRT, intensity modulated radiotherapy; Al, aluminum; POP, plaster of paris; RT, radiation therapy

\section{Introduction}

External Beam Radiotherapy (EBRT) remains the mainstay for radical treatments, in malignancies in head and neck regions. Teletherapy with cobalt-60 isotope machines are still available for cancer management in countries with more population, and their continuation as a basic machine has been recommended. ${ }^{1}$ With linear accelerators, the present day radiotherapy for radical treatments in head and neck are to use Intensity modulated radiotherapy (IMRT) to limit doses to unilateral or bi-lateral parotid, sub-mandibular glands, and to limit spinal cord doses. Because of less inter-field separations, the penetration requirements of the treatment plans makes the tele cobalt beam sufficient for executing radiotherapy, with proper immobilization and use of wedges if required.
The tissue thickness and irregular contour variations encountered in these patients affect uniformity in radiation dose delivery. Adverse reactions are encountered because of skewed isodose curves, seriously affecting the volume dose variations in treatment plans. This complex situation results in dose inhomogeneity, resulting in more skin reactions in the head and neck region, often discouraging the patient from taking full course of treatment. The above issues also relate to adjuvant surgical management when the texture integrity of skin in neck region is not preserved. Non-uniformity of dose adversely influence normal tissue reactions, affecting effectiveness of the treatment and therefore deter the Quality Of Life (QOL) in the follow up period.

Cobalt- 60 beam quality has a maximum absorbed dose built up at $5 \mathrm{~mm}$ down the entrance field, and any tissue compensation done at the surface affect the build up effect, bringing dose to $100 \%$ at skin level and therefore should be avoided. Ellis type compensator ${ }^{2,3}$ use aluminum (Al) square pellets, kept far away from the skin in the path 
of the beam. The compensator plates are custom made for individual radiotherapy portals. Tissue deficiencies are addressed by fixing small thickness $\mathrm{Al}$ rods taking density factor. Commercially available jigs for fabrication of aluminum tissue compensators do not account for beam divergence at the collimator level. However, if the width of square rods are taken to account for divergence representing larger squares at skin level then the error involved will be insignificant Divergence type compensators using wax instead of metals are recently reported, 4,5 which use a special jig to take impression, and preparing wax irregular prosthesis at the tray level. In their methodology, the need for a positive Plaster of Paris (POP) impression of head and neck is mentioned.

Radiation Oncology Department at Cachar Cancer Hospital and Research Centre (CCHRC) started external radiation therapy (RT) treatments with an imported telecobalt machine (Theratron 780C) during 2006 and completed a decade. The department is in the process of upgrading by adding a linear accelerator with precision treatments, in the near future. The representative patient's distribution treated by RT CCHRC is shown in Table 1. Almost nearing $50 \%$ of the total cases receive RT for Head and Neck region. The telecobalt machine has only rectangular X and Y Collimators, and no penumbra trimmers.

Table I Number of cases treated by radiotherapy at $\mathrm{CCHRC}$ and site wise distribution for 3years

\begin{tabular}{|c|c|c|c|c|c|c|c|}
\hline No. & Site & 2013 & 2014 & 2015 & $2013 \%$ & $2014 \%$ & $2015 \%$ \\
\hline I & H \& Neck & 170 & 173 & 184 & 51.1 & 46.9 & 48.4 \\
\hline 2 & Ca.Cervix & 35 & 32 & 51 & 10.5 & 8.7 & 13.4 \\
\hline 3 & Ca.Breast & 27 & 42 & 28 & 8.1 & 11.4 & 7.4 \\
\hline 4 & Ca.Oesoph & 20 & 24 & 38 & 6 & 6.5 & 10 \\
\hline 5 & Ca.Rectum & 12 & 21 & 20 & 3.6 & 5.7 & 5.3 \\
\hline 6 & Ca.Lung & 9 & 9 & 13 & 2.7 & 2.4 & 3.4 \\
\hline 7 & Brain Tum & 9 & 14 & 18 & 2.7 & 3.8 & 4.7 \\
\hline 8 & Metastases & 23 & 21 & 10 & 6.9 & 5.7 & 2.6 \\
\hline 9 & Ca.Vagina & 4 & 2 & 3 & 1.2 & 0.5 & 0.8 \\
\hline 10 & Ca.Uri Blad & I & I & 1 & 0.3 & 0.3 & 0.3 \\
\hline 11 & Ca.Prostate & 1 & 2 & I & 0.3 & 0.5 & 0.3 \\
\hline 12 & Sarcomas & 2 & 2 & ---- & 0.6 & 0.5 & ----- \\
\hline 13 & Lymphoma & 3 & -- & I & 0.9 & ---- & 0.3 \\
\hline \multirow[t]{2}{*}{14} & Others & 17 & 26 & 12 & 5.1 & 7 & 3.2 \\
\hline & Total & 333 & 369 & 380 & & & \\
\hline
\end{tabular}

The problem in dosimetry of head and neck regions is, when chin level maximum thickness is taken for planning, the chin and neck level mid plane doses become high. Also the skin and subcutaneous tissues get overdosed, wherever the contour thickness is less. To reduce skin reactions, and to maintain uniform doses to mandible and neck areas, we recently introduced tissue compensation to account thickness variations when RT field encompass both head and neck region. Divergent type wax tissue compensators have been reported in literature recently. ${ }^{4}$ As the hardware for preparing them is not available readily as well as commercially, we resorted to making Aluminum Tissue compensators. The implementation of the overall process is outlined in this report.

\section{Materials and methods}

\section{Patients selection}

We plan to prepare Al tissue compensators for all the patients planned for radical treatments. Initially, only true lateral irradiation treatments will have $\mathrm{Al}$ compensators kept in the path of the beam. At a later stage when more patients have been completed, AP, PA compensators also will be prepared. Patients will be explained about the need for taking separate measurements of tissue deficits using special calipers prepared for this purpose. Iso dose distributions for the plan will be prepared if requested by the radiation oncologist.

\section{Measurements for the patient}

Inter-field separation at Mouth/Chin level contour is obtained for dose calculation at thickest separation. Orfit thermo-plastic cast is not used for these patients because of setting up lateral fields with shadow tray frame mounting on the machine. Instead an immobilization Plaster of Paris (POP) cast will be prepared, with patient having a shoulder retractor 'on'. At $1.5 \mathrm{~cm}$ intervals, throughout the planned RT field size, the tissue deficits will be recorded using a special type Perspex caliper (Figure 1), keeping the patient to maintain a fixed Sternal Notch to Chin distance (NC). During measurement, the immobilization Plaster of Paris (POP) will not be existing. The caliper used will be of 'L type" Perspex Mount on which patient is positioned with the neck rest. The depth values are noted down for all the grid of points; the tissue deficiency is calculated by seeing difference between minimum depth to individual depths.

\section{Preparation of the $\mathrm{Al}$ compensator}

The compensator Al square cross section rods are of dimension $10 \mathrm{~mm}$ of varying lengths from $2 \mathrm{~mm}$ to $6 \mathrm{~cm}(\mathrm{M} / \mathrm{s}$ Meditronix Corporation, Delhi). The tissue deficiency readings are converted into Aluminum thickness based on $\mu=0.1462 \mathrm{~cm}^{-1}$ measured for $20 \times 20$ field measured by keeping them at tray level (corresponding to $1 \mathrm{~cm}$ $\mathrm{Al}=2.57 \mathrm{~cm}$ water). As type of material content of commercially 
available Aluminum rods may not be pure, the value of $\rho=2.7 \mathrm{gm} / \mathrm{cc}$ factor is not used. The type of $\mathrm{Al}$ rods used is shown in Figure 2 and assembled $\mathrm{Al}$ Compensator mount for a patient is shown Figure 3. Chin level and neck level contour of the patient taken with POP strip is shown in Figure 4.

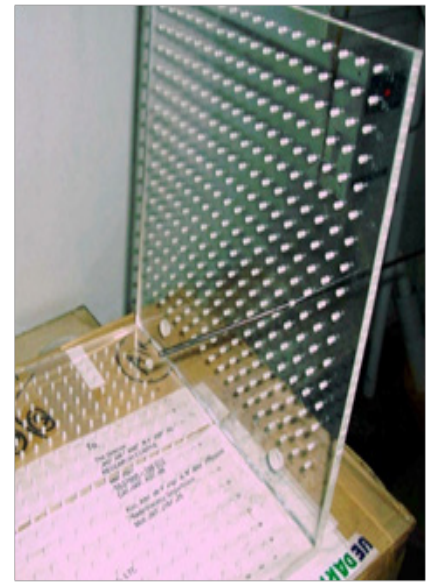

Figure I Special type perspex caliper.

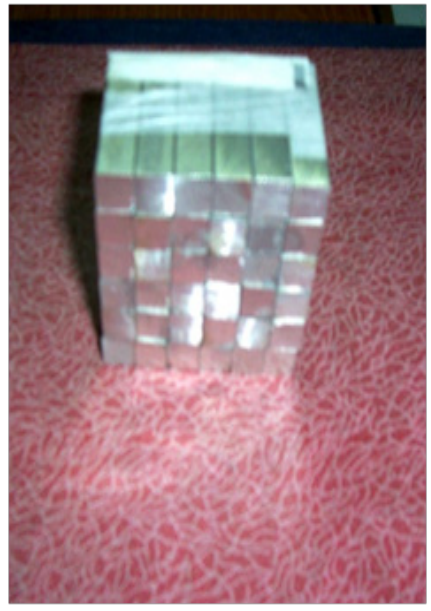

Figure 2 Type of Al rods.

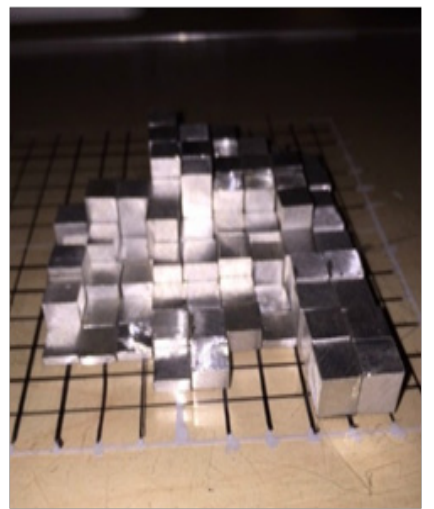

Figure 3 Assembled Al compensator mount for a patient.

\section{Type of immobilization}

As Orfit cast is not being used for the patient, a simple type of POP immobilization is prepared by having a POP strip at the chin and another at shoulder level. The patient positioned with this immobilizer in cobalt machine is shown in Figure 5. The patient with shoulder retractor in position along with mounted $\mathrm{Al}$ Compensator on the treatment head of cobalt machine is shown in Figure 6.

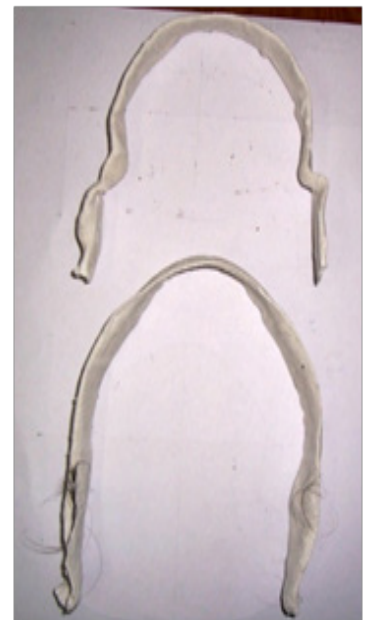

Figure 4 patient with POP strip.

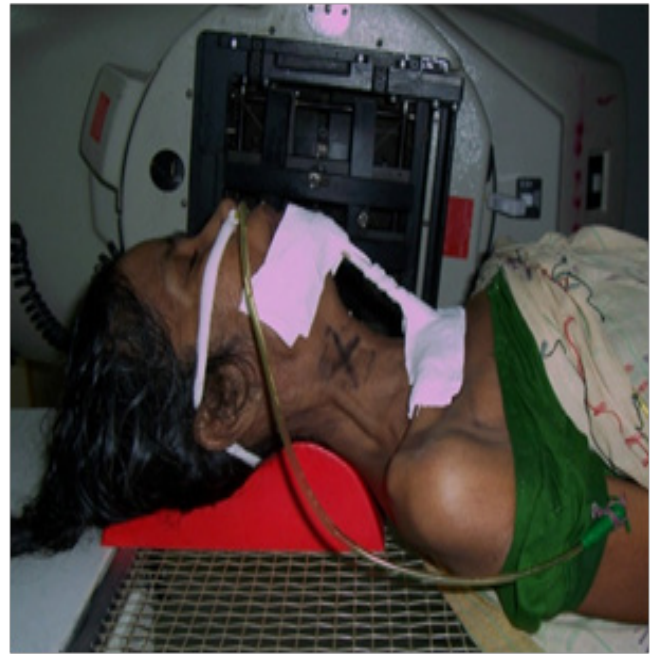

Figure 5 POP neck immobilization.

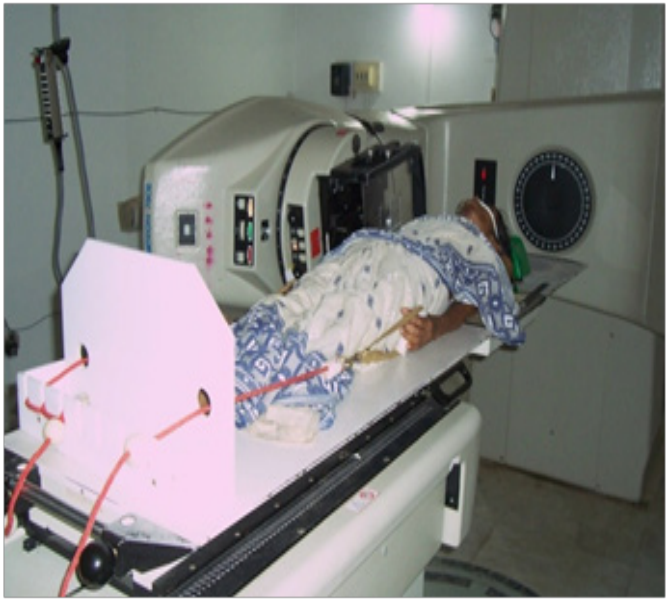

Figure 6 Patient positioned with arm retractor. 


\section{Treatment time calculation}

The calculated dose is for maximum inter-field separation, because the metal compensator corrects for the tissue deficiency. The treatment time is calculated to larger separation, and therefore about $10 \%$ excess source 'ON' time. The mounting plate is made up of Perspex, which attenuates the beam by $4 \%$ and that correction is also applied during time calculation.

\section{Portal films}

A verification of the penetrated exit dose pattern without compensator is acquired by having digital $\mathrm{CR}$ cassette exposed to $0.03 \mathrm{~min}(1.8 \mathrm{sec})$ for smaller port followed by open big field $2^{\text {nd }}$ exposure for $0.03 \mathrm{~min}(1.8 \mathrm{sec})$. With compensator inserted in the beam another port film was taken for double the time.

\section{Results}

\section{Efficacy of the treatment technique}

Homogenization in delivered dose in head and neck region: By using this custom type $\mathrm{Al}$ compensators, homogeneity in dose in achieved within $2-3 \%$. Figure 7 shows the isodose distributions at mouth level and mid plane of neck level without and with $\mathrm{Al} \mathrm{Com-}$ pensators. It is seen that in the neck region $180 \%$ dose line is reduced to $150 \%$ and in the chin region $170 \%$ dose line is reduced to $150 \%$; at the same time $160 \%$ covering the central tumor volume adequately. This is in line with an overdose pattern to the neck and chin area as high as $17 \%$ with plain field reported in literature earlier. ${ }^{5}$

The port films taken without (Figure 8A) and with compensator (Figure 8B) clearly showed good homogenization achieved by the use of Aluminum compensator.

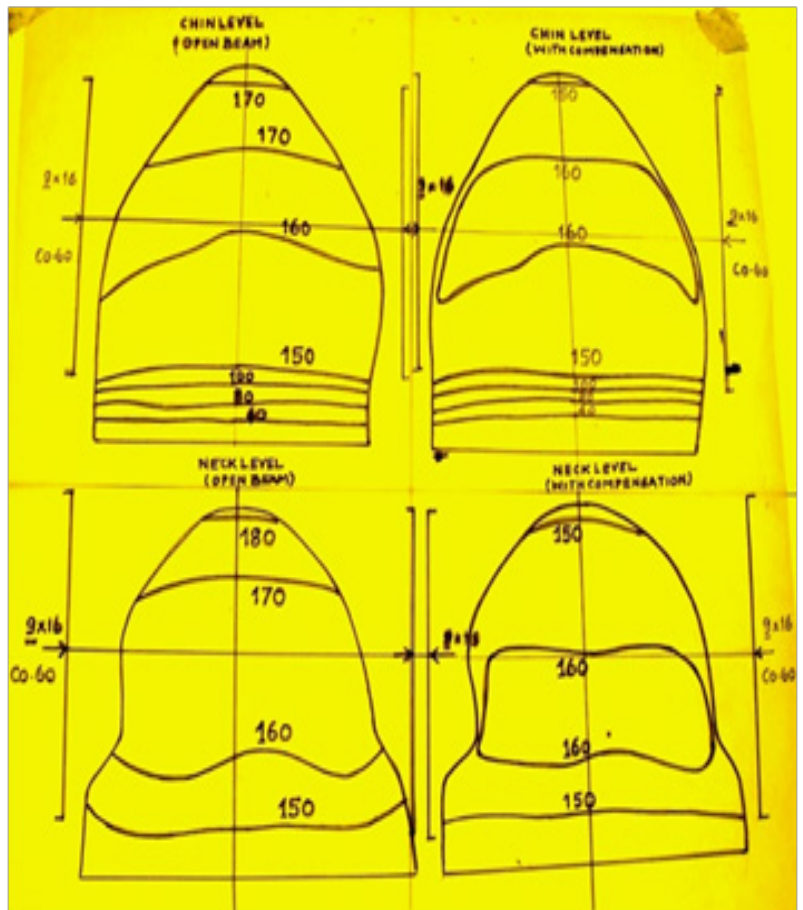

Figure 7 Is dose distributions at mouth and neck levels. (Left side up and down distributions without compensator).

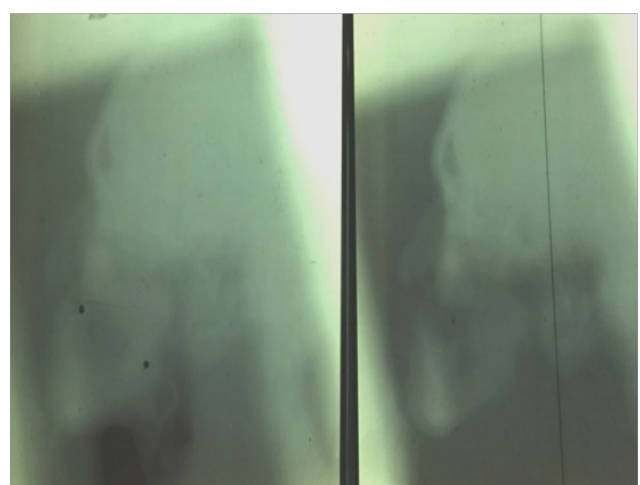

Figure 8 (A) Port film with open field (B) Port film with compensator (arrow shows nor-uniform density hot spot in neck area).

\section{Discussion}

This work clearly brought out the steps involved in the introduction of custom built $\mathrm{Al}$ compensators for head and neck radiation therapy. Though it is well established and explained ${ }^{3}$ method, this is not widely practiced because of the involved intricacies in measurements and assembling on acrylic sheets. In the era of modern linear accelerators, this type of introduction of methods brings better dose delivery even with a simple telecobalt machine. Long time experience in India more than 5 decades, in the use of telecobalt megavoltage radiotherapy for head and neck regions for radical treatments, adds in support of these innovations which could be cost effective. We have substituted thermoplastic immobilization with a simple POP chin support which gives similar patient comfort. Moreover, the need for cutting open the thermoplastic molds for field entries (to preserve dose build up) is obviated by using open type POP chin support.

An evaluation of the efficacy of the custom built compensator had been reported in literature..$^{5}$ In this a phantom dosimetric study was carried out with Aluminum tissue compensators (TC) using GaF chromic film, ion chamber and thermo-luminescent dosimeter. For compensated and uncompensated plans they found the following observations. For open-field plans, peak doses were as high as $117 \%$, optimally wedged-plans contained peak doses of $113 \%$, and customcompensated plans contained peak doses of $105 \%$. The dose variation between prescribed and measured dose at mid plane of the phantom was as high as $17 \%$, which was reduced to $3.2 \%$ for the customized TC during ionometric measurements. In the sagittal plane, it was evaluated with TLDs that the high-dose region of $13.3 \%$ was reduced to $2.3 \%$. Thus, a custom-made $2 \mathrm{D}$ TC is capable of reducing hot spots to improve overall dose homogeneity across the treatment volume.

The isodose patterns compared for with and without compensators at Chin and Neck levels (Figure 7) clearly show similar observation. Tip of the chin had $170 \%$ iso dose line reducing to $150 \%$ with compensation; and tip of the neck contour receiving 180\% dose with open field reducing to $150 \%$. In both the sections, the central portion having same coverage line of $160 \%$ clearly bringing out the effect of tissue compensation. The work by Srinivas et al. ${ }^{6}$ outlined the comparison of skin texture in the patients treated with wax divergent tissue compensator, bringing out the need for application of tissue compensation in head and neck radiotherapy. As the energy of the photon beam increases, the inhomogeneity in parallel opposed fields slightly reduce. However, the introduction of compensators 
will definitely have advantageous effect for 6MV linac beams also, which are commonly used for head and neck treatments presently. In Figure 8 we have brought out the portal film pattern different with compensation showing good uniformity of dose. We observed in about 12 patients so far treated no skin reactions, and therefore up to a dose of 70Gy the tumor volume could be treated without any break in treatment. All patients prefer this treatment showing better acceptance with least morbidity. In most of the developing countries, still cobalt machine is the machine of choice. In the north eastern parts of India, and Ban gala Desh, cancers of head and neck are the common cancers, and therefore the method described in this work could help the therapy centres, as an easy solution. Though it is a bit labor intensive in terms of man hours to assemble the custom built tissue compensator, this is worth because in head and neck cancers, radiotherapy is the primary modality. The skin texture preservation helps the surgeons to intervene at a later stage if there are developed recurrences.

There are some precautions need to be observed by the technologists during the use of compensators. The suggested precautions are:

a) Patient's position should be reproduced.

b) Orientation of the Compensator Assembly during insertion in shadow tray, very important.

c) As we use the same compensator insert for both lateral beams, its orientation needs reversal. If wrongly placed, there will be under-dosage to thick area, overdosing to thin area.

d) It shall be always remembered, that the time of irradiation is calculated for the thickest separation of the patient (because deficient thickness are compensated by Al metal thickness). If the compen- sator plate is not mounted, it will end up with an overdose as high as about $15 \%$ due the excess time which takes into account of the extra attenuation in the path of the beam.

\section{Acknowledgements}

The authors thank Director, CCHRC for kind permission for this communication. Head and Neck Radiotherapy with Tele-cobalt machine- Efficacy and need for tissue compensation.

\section{Conflict of interest}

Author declares that there is no conflict of interest.

\section{References}

1. Ravichandran R. Has the time come for doing away with Cobalt-60 teletherapy for cancer treatments. J Med Phys. 2009;34(2):63-65.

2. Ellis F, Hall EJ, Oliver R. A compensator for variations in tissue thickness for high energy beams. Br J Radiol. 1959;32(378):421-422.

3. Khan FM. The Physics of Radiation Therapy. 4th ed. USA: Lippincott, Williams, Wilkins Pubs; 2010.

4. Srinivas C, Shenoy K, Vidya K, et al. Wax Tissue Compensator for Head and Neck Radiotherapy - Fabrication and Dosimetric Evaluation. J Cancer Res Ther Oncol. 2015;3:1-5.

5. Kinhikar RA, Tambe CM, Upreti RR, et al. Phantom dosimetric study of non-divergent aluminum tissue compensator using ion chamber, TLD, and gafchromic film. Med Dosim. 2008;33(4):286-292.

6. Srinivas C, Shenoy K, Shenoy V, et al. Fabrication of Divergent Wax based compensators for Head and Neck Radiotherapy. Med Phys. 2011;38:3394. 